\title{
Effect on coupling synchronization of Morris-Lecar neurons under time-delay.
}

\author{
Ruining Fan ${ }^{1 *}$, Boping Zhang ${ }^{2}$ \\ ${ }^{1}$ School of Information Engineering, Lanzhou University of Finance and Economics, China \\ ${ }^{2}$ College of Information and Engineering, Xu Chang University, China
}

\begin{abstract}
Based on the modified Morris-Lecar neuron mathematical model, this paper conducts the numerical calculation and simulation of the Morris-Lecar neuron mathematical model and studies the synaptic coupling synchronization of Morris-Lecar neurons under the effect of time-delay through the phase plane portrait, the time response graph and the synchronization difference graph. It is shown that the time-delay can promote the synchronization of the asynchronous coupled Morris-Lecar neurons and induce the coupling synchronization of the neurons, which illustrates time-delay has a certain effect on coupling neuronal system.
\end{abstract}

Keywords: Morris-Lecar neuron model, Coupling synchronization, Time-delay.

Accepted on May 23, 2017

\section{Introduction}

The nervous system is an organ system which is composed of a network of neurons, a kind of specialized cell. The nervous system can sense the external stimuli. Time-delay is time delay, and its appearance triggers more complex nonlinear dynamic characteristics, so the influence of time delay on nonlinear dynamical behavior of the coupled neuron system has attracted more attention. With the deepening of research, neural networks such as time-delay Hopfield neural network [1], delayed bidirectional associative memory neural network [2], time-delay cellular neural network and time-delay Cohen-Grossberg [3] are put forward successively. The neural coupling system with time delay function also attracts a large number of experts and scholars at home and abroad to study and explore, and many meaningful results have been achieved [4,5]. This paper introduces the neuron model and related knowledge, introduces the related biological knowledge of the nervous system and the related techniques used in this paper. This paper establishes a Morris-Lecar chaotic neuron model with two neuronal electrical synapse couplings makes a dynamic analysis of the MorrisLecar neuron model by numerical simulation and simulation and studies the effect of the electric synaptic coupling intensity of Morris-Lecar neuron model on the generation and release of electrical activity and synchronization of the system.

In this paper, the synchronization behavior of two neuron systems with time-delayed electrical synapse coupling is established. The dynamical behavior of the system is described by the following time-delayed differential equations:

$$
\left\{\begin{array}{l}
\frac{d V_{1}}{d t}=-u_{1}-g_{l}\left(V_{1}-V_{l}\right)-g_{k} w_{1}\left(V_{1}-V_{k}\right)-g_{c a} m_{\infty}\left(V_{1}\right)\left(V_{1}-V_{c a}\right)+D\left(V_{2}(t-\tau)-V_{1}\right) \\
\frac{d w_{1}}{d t}=\lambda\left(V_{1}\right)\left(w_{\infty}\left(V_{1}\right)-w_{1}\right) \\
\frac{d u_{1}}{d t}=\mu\left(0.2+V_{1}\right) \\
\frac{d V_{2}}{d t}=-u_{2}-g_{l}\left(V_{2}-V_{l}\right)-g_{k} w_{2}\left(V_{2}-V_{k}\right)-g_{c a} m_{\infty}\left(V_{2}\right)\left(V_{2}-V_{c a}\right)+D\left(V_{1}(t-\tau)-V_{2}\right) \\
\frac{d w_{2}}{d t}=\lambda\left(V_{2}\right)\left(w_{\infty}\left(V_{2}\right)-w_{2}\right) \\
\frac{d u_{2}}{d t}=\mu\left(0.2+V_{2}\right)
\end{array}\right.
$$

In the equations above, the values of system parameters are as follows: $V_{c a}=0.84, V_{l}=-0.5 \mathrm{mV}, V_{k}=-0.87 \mathrm{mV}, v_{1}=-0.01 \mathrm{mV}$ $, v_{2}=0.15 \mathrm{mV}, \quad g_{l}=0.5 \mu \mathrm{F} / \mathrm{cm}^{2}, g_{k}=2 \mu \mathrm{F} / \mathrm{cm}^{2}, \quad g_{c a}=1.2 \mu \mathrm{F} / \mathrm{cm}^{2}$. $D$ represents the coupling strength, $\tau$ is on behalf of the ML neuronal information transmission delay, $w$ is on behalf of the recovery variable. $m_{\infty}(V)$ and $w_{\infty}(V)$ represent the steadystate values of the open probability of the $\mathrm{Ca}^{2+}$ and $\mathrm{k}^{+}$channels respectively.

First, we study the synchronization of the neuron system without time-delay. Let t $D=0.001,\left(V_{1}, V_{2}\right)$, and the phase plane portrait of the two electric synapse coupled ML neurons on the $\left(V_{1}, V_{2}\right)$ plane can be obtained by the differential equation, which is shown as Figure 1.

It can be seen from Figure 1, that the system is in an asynchronous state at this time and from Figure 2, that the values of $V_{1}$ and $V_{2}$ are different when they are at the same time, indicating that the two neurons do not reach the synchronized state. In order to better observe whether the two nerves are in the synchronous state or not, the introduction of neuron-coupled synchronization difference map is also made, as shown in Figure 3. It can be clearly seen from Figure 3 that the system is in the nonsynchronous state and the system is in a periodic discharge activity state but not synchronized.

From the above analysis, it can be concluded that, within a certain range, the time delay can enhance the synchronization of the electrical synapse-coupled Morris-Lecar neurons and promote the coupling neurons to transfer from the asynchronous discharge patterns to the fully synchronous discharge patterns.

\section{Conclusion}

This paper studies the synergistic enhancement of electrical synaptic-coupled Morris-Lecar neuron system with timedelay. The results show that the appropriate time delay can enhance the occurrence of electrical synapse coupling neurons synchronization. These study results have important 


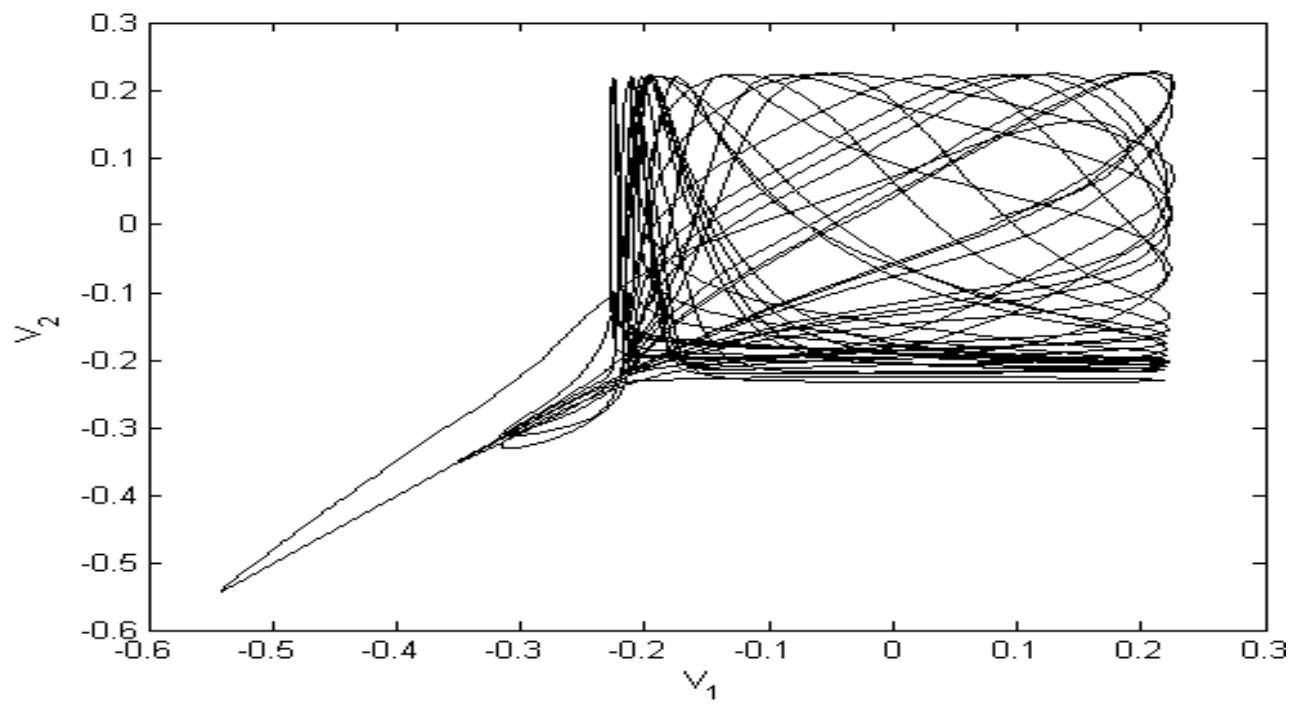

Figure 1. Phase plane graph when and $\tau=0$.

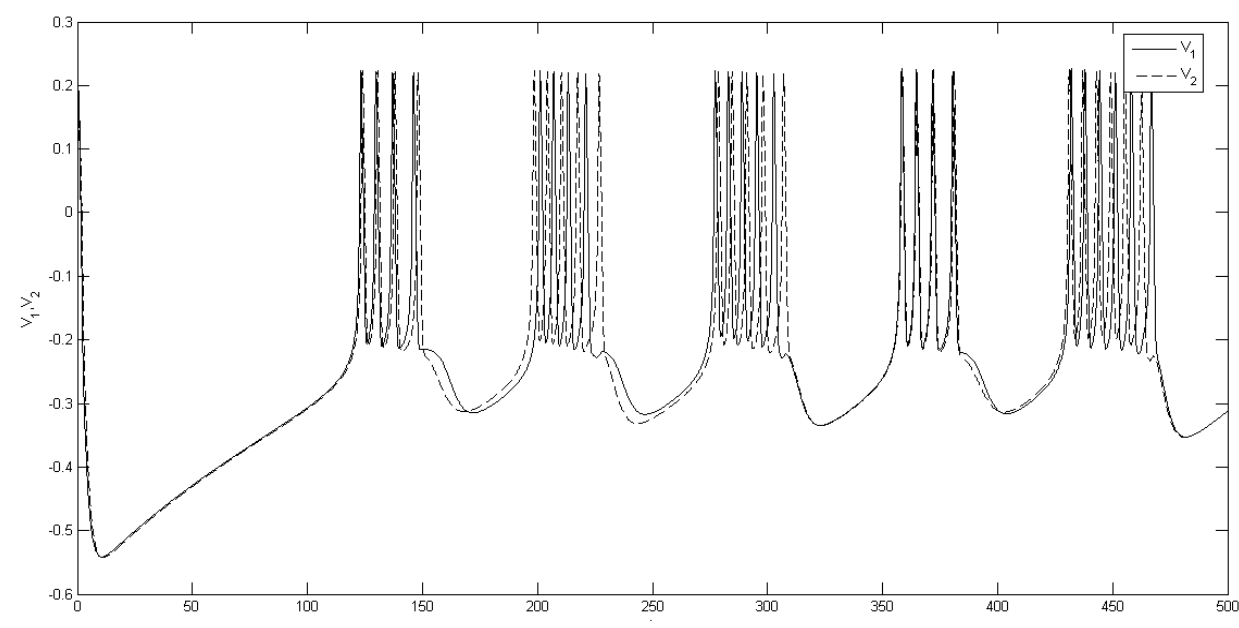

Figure 2. Time response diagram when $D=0.001$ and $\tau=0$.
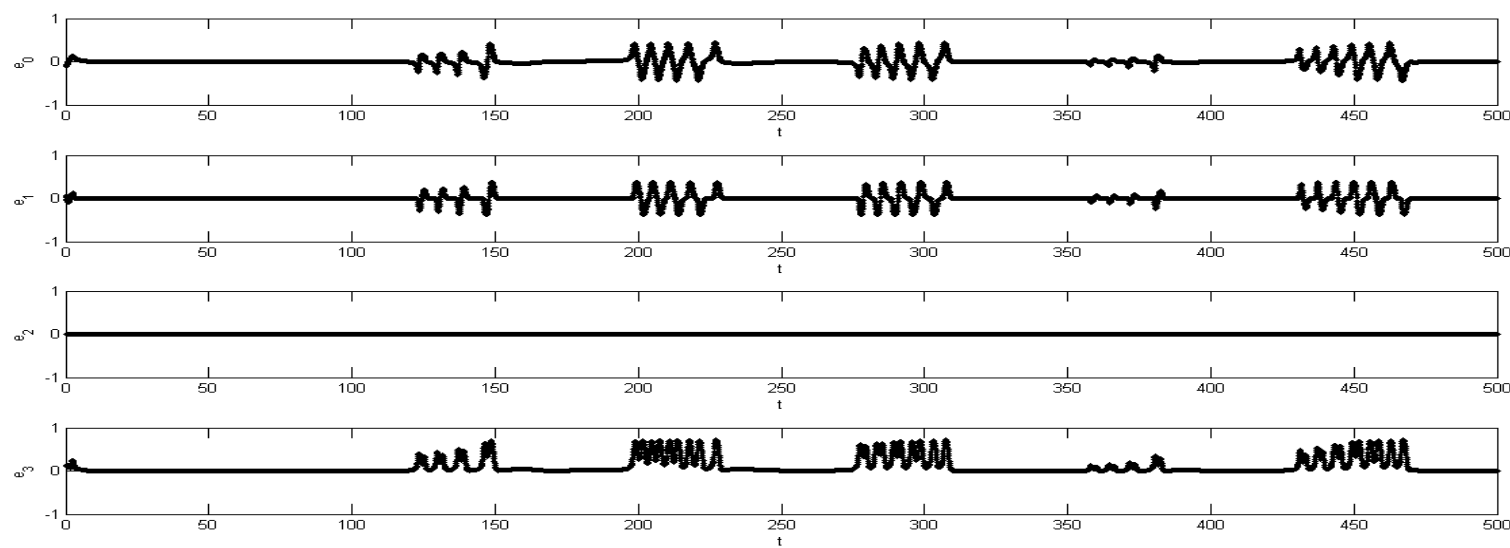

Figure 3. Synchronization difference graph when $D=0.001$ and $\tau=0$. 
implications for the further understanding of the effects of time delay on the discharge pattern of the coupled neuron system.

\section{Acknowledgement}

This work was financially supported by the Scientific Research Project of Colleges and Universities in Gansu Province (No.2016B-051).

\section{References}

1. Mou S, Gao H, Lam J, et al. A new criterion of delaydependent asymptotic stability for hopfield neural networks with time delay. IEEE Transactions on Neural Networks. 2008;19(3):532-5.
2. Sakthivel R, Vadivel P, Mathiyalagan K, et al. Design of state estimator for bidirectional associative memory neural networks with leakage delays. Inform Sci. 2015;296:263-74.

3. Ke Y, Miao C. Stability analysis of fractional-order CohenGrossberg neural networks with time delay. Int J Comput Math. 2015;92(6):1102-13.

4. Burić N, Ranković D, Todorović K, et al. Mean field approximation for noisy delay coupled excitable neurons. Physica A Stat Mech Appl. 2010;389(19):3956-64.

5. Zhen B, Xu J. Simple zero singularity analysis in a coupled Fitzhugh-Nagumo neural system with delay. Neurocomputing. 2010;73(4-6):874-82.

\section{*Correspondence to:}

School of Information Engineering

Lanzhou University of Finance and Economics China

Tel: +86-0931-4938613

E-mail: 476266852@qq.com 